\title{
LINFOMA PLASMABLÁSTICO EM PACIENTE COM INFECÇÃO PELO VÍRUS DA IMUNODEFICIÊNCIA HUMANA
}

\author{
PLASMABLASTIC LYMPHOMA IN PATIENT WITH INFECTION BY THE HUMAN IMMUNODEFICIENCY VIRUS
}

lago Barbosa Pinto Rodrigues, Gabryel Cordeiro de Lima, Marta Rodrigues Félix, Lara de Paula Sousa, Rayanne Garrido Monteiro de Andrade

DOI - 10.5935/2236-5117.2019v56a10

\section{RESUMO}

O linfoma plasmablástico é um tumor raro que corresponde a $2 \%$ dos linfomas não-Hodgkin (LNH). É importante ampliar o conhecimento e os possíveis desfechos a respeito dessa condição visto que há escassez de estudos. Relata-se o caso de uma paciente com infecção pelo vírus da imunodeficiência humana (HIV), que vinha há 10 anos em uso regular de antirretrovirais. Há 1 ano iniciou quadro de hiporexia, perda ponderal, astenia e lesões aftosas em mucosa oral, que se associaram a episódio de síncope. A investigação clínica subsequente confirmou diagnóstico de linfoma plasmablástico. Foi submetida a quimioterapia, inicialmente refratária. Prosseguiu com mais 2 ciclos quimioterápicos, com resposta satisfatória confirmada por controle radiológico. Após 6 meses, apresentou sintomas inespecíficos associados a sinais neurológicos. Realizou ressonância magnética de crânio que evidenciou acometimento metastático cerebral sendo, então, indicado cuidados paliativos.

Palavras-chave: Linfoma plasmablástico; linfoma não Hodgkin; síndrome de Imunodeficiência adquirida.

\section{ABSTRACT}

Plasmablastic lymphoma is a rare tumor that accounts for $2 \%$ of non-Hodgkin's lymphomas (NHL). It is essential to increase the knowledge and possible outcomes regarding this condition since studies are scarce. We report a patient with infection by the human immunodeficiency virus (HIV) for ten years on the regular use of antiretrovirals. One year ago she started hyporexia, weight loss, asthenia, and aphthous lesions in the oral mucosa that associated with an episode of syncope. The subsequent clinical investigation confirmed a diagnosis of plasmablastic Iymphoma. She underwent chemotherapy, which was refractory. She continued with two more chemotherapy cycles, with satisfactory response confirmed by radiological control. After six months, she developed nonspecific symptoms associated with neurological signs. The patient underwent magnetic resonance imaging of the skull that showed metastatic brain involvement, and then palliative care was indicated

Keywords: Plasmablastic Iymphoma; Iymphoma, nonHodgkin; acquired immunodeficiency syndrome. lago Barbosa Pinto Rodrigues - Rodrigues IBP. Acadêmico de Medicina. Curso de Graduação em Medicina, Escola Superior de Ciências da Saúde (ESCS), Brasília, Distrito Federal, Brasil.

Gabryel Cordeiro de Lima - Lima GC. Acadêmico de Medicina. Curso de Graduação em Medicina, Escola Superior de Ciências da Saúde (ESCS) Brasília, Distrito Federal, Brasil.

Marta Rodrigues Félix - Félix MR. Acadêmica de Medicina. Curso de Graduação em Medicina, Escola Superior de Ciências da Saúde (ESCS), Brasília, Distrito Federal, Brasil.

Lara de Paula Sousa - Souza LP. Médica. Unidade de Clínica Médica, Hospital Regional de Taguatinga, Brasília, Distrito Federal, Brasil.

Rayanne Garrido Monteiro de Andrade - Andrade RGM. Médica. Unidade de Clínica Médica, Hospital Regional de Taguatinga, Brasília, Distrito Federal, Brasil.

Correspondência: lago Barbosa Pinto Rodrigues. Curso de Graduação em Medicina, Escola Superior de Ciências da Saúde. SMHN Quadra 03, conjunto A, Bloco 1 Edifício FEPECS, Brasília, Distrito Federal, Brasil. CEP 70.710-907.

Internet: iagoprodrigues15@gmail.com

Conflito de interesses: Não há potenciais conflitos a declarar.

\section{INTRODUÇÃO}

Os linfomas não Hodgkin (LNH) são o segundo tipo de câncer mais frequente em pacientes portadores do vírus da imunodeficiência humana positivos (HIV). ${ }^{1}$ Entre os LNH, o linfoma plasmablástico corresponde a menos de $5 \%$ dos casos. ${ }^{2,3}$

Em pesquisa com o termo "Iinfoma plasmablástico" na Scielo foram encontradas sete publicações, sendo seis relatos de caso e um artigo de revisão. Na base de dados LILACS, sete publicações. Na PUBMED, usando o termo de pesquisa "plasmablast lymphoma", foram encontrados cinco relatos de caso, nenhum relacionado a paciente com infecção pelo HIV.

Olinfoma plasmablástico representauma porcentagem de $2,6 \%$ em pacientes com sorologia positiva para HIV e com diagnóstico de linfoma associado. ${ }^{4}$ 
Dada a escassez de literatura, relatou-se o caso clínico de uma paciente do sexo feminino com infecção pelo HIV e diagnóstico confirmado de linfoma plasmablástico.

\section{RELATO DO CASO}

Paciente de 39 anos, brasileira, sabidamente com infecção pelo HIV há 10 anos, em uso regular de terapia antirretroviral com dolutegravir, emtricitabine e tenofovir, desde o diagnóstico. Evoluiu, há um ano, com perda ponderal, astenia e lesões aftosas em mucosa oral, que se associaram a episódio de síncope, motivando a investigação clínica que evidenciou o diagnóstico de LNH do tipo plasmablástico confirmado por biópsia em nódulo na nádega.

Foi submetida, então, a seis ciclos do esquema de poliquimioterapia EPOCH (etopósido, prednisona, oncovinm, ciclofosfamida e hidroxidaunorubicina), associado a quatro ciclos de metotrexato em altas doses. Todavia, apresentou refratariedade ao tratamento, constatado em exame histopatológico de um novo nódulo em nádega direita. Foram necessários mais 2 ciclos de VTD-PACE (bortezomibe, talidomida, dexametasona, cisplatin, doxorubicin, cyclophosphamidee etoposide). No controleradiológico após a quimioterapia, foi realizada tomografia por emissão de pósitrons (PET), que evidenciou resposta completa ao esquema terapêutico instituído.

Após 6 meses, evoluiu subitamente com paraplegia, confusão mental, estrabismo súbito, febre e vômitos. Foi submetida à exame de ressonância magnética (RNM) de encéfalo, que evidenciou metástases multissistêmicas, incluindo o sistema nervoso central, com acometimento leptomeningeal difuso, lesão de $9 \mathrm{~mm}$ com realce no hemisfério cerebelar direito e realce patológico dos nervos facial e vestibulococlear.

Diante dos novos achados radiográficos, optou-se pela indicação de cuidados paliativos e a paciente retornou ao país natal em busca de suporte familiar.

\section{DISCUSSÃO}

O linfoma plasmablástico, em meio aos outros tipos de LNH, é a manifestação linfoproliferativa com menor incidência, sendo quase exclusiva em pacientes com infecção pelo HIV. ${ }^{1}$

A frequência com que ocorrem os LNH é diretamente proporcional ao avanço natural da imunodepressão e da redução da contagem de linfócitos CD4 secundários à infecção pelo HIV. Ademais, apesar do advento da terapia antirretroviral, a incidência desse tipo de câncer não reduziu. ${ }^{1}$
Nos pacientes com síndrome da imunodeficiência adquirida (SIDA) e linfoma, notam-se niveis aumentados de CD23 solúvel quando comparado aos pacientes com SIDA e que não desenvolvem linfoma, com sorologia positiva para HIV sem SIDA, e pessoas sem HIV. Isso ocorre devido a estimulação crônica que a infecção pelo HIV causa nas células $B$, à redução da vigilância imunológica e às lesões genéticas de caráter oncogênico. Desse modo, a mitogênese propiciada pelos estímulos crônicos amplia a mutação genética e propicia o desenvolvimento da neoplasia. ${ }^{1}$

O termo "plasmablástico" é utilizado devido ao fato desses linfomas exibirem a morfologia dos imunoblastos, mas expressarem perfil antigênico de plasmócitos. Como não são observados vírus HIV nas células do linfoma plasmablástico, não se pode conferir o surgimento dessa doença exclusivamente à presença do HIV. Entretanto, a imunossupressão causada pelo vírus propicia a infecção por outros vírus conhecidamente oncogênicos, como os vírus Epstein Barr5, que podem ser detectados em $74 \%$ dos pacientes com linfoma plasmablástico associado ao HIV. ${ }^{6}$

As queixas mais comuns do linfoma plasmablástico são emagrecimento, sudorese noturna e febre. ${ }^{1}$ Porém, os sintomas geralmente só se manifestam em estágios avançados da doença, com metástases acometendo diversos órgãos e sítios externos ao sistema linfático (característica encontrada em 75 a 90\% dos casos). No momento do diagnóstico, já há acometimento meníngeo em 15 a $20 \%$ dos casos, e $80 \%$ dos pacientes já se encontram no estágio IV da doença. As manifestações clínicas mais comuns nesse estágio incluem radiculalgia, dorsalgia, cervicalgia e cefaleia, sendo outras manifestações menos comuns: paralisia de nervo craniano, alterações no estado mental, perda sensorial e fraqueza. Em torno de 25\% dos pacientes apresentam acometimento de medula óssea, geralmente associado a um quadro de plaquetopenia, histologia de Burkitt ou Burkitt-like, envolvimento leptomeníngeo, bem como ao aumento da desidrogenase láctica (DHL). Somente $25 \%$ dos pacientes encontram-se assintomáticos ao diagnóstico. ${ }^{7}$

O exame de punção liquórica é essencial para o estadiamento da doença, pois cerca de 35\% dos pacientes, ao longo do seguimento da doença, desenvolvem alguma sintomatologia meníngea. ${ }^{1}$ Aproximadamente $25 \%$ dos pacientes com LNH-HIV e acometimento leptomeníngeo são assintomáticos. ${ }^{8}$

Pacientes com sorologia para HIV que desenvolvem LNH apresentam prognóstico reservado com sobrevida média após o diagnóstico de cerca de 1 ano. Embora, na França, tenha sido relatada uma estimativa de sobrevida em 5 a 10 anos de 41 a $48 \%$ dos casos de pacientes com SIDA e diagnóstico 
de linfoma primário no sistema nervoso central, as estimativas na população geral são mais modestas. Em uma análise do banco de dados do National Cancer Center, nos Estados Unidos, a sobrevida global média destes pacientes foi de 3,6 meses. ${ }^{9}$ Em torno de $50 \%$ dos pacientes com LNH e infecção pelo HIV vão a óbito devido a infecções oportunistas, e os outros $50 \%$ devido a progressão ou recidiva da doença linfocitária.

Os fatores de mau prognóstico incluem diagnóstico prévio de SIDA, nível de linfócitos CD4 abaixo de $100 / \mathrm{mm}^{3}$, utilização de drogas injetáveis, idade superior a 35 anos, estágio avançado da doença, DHL elevada, linfoma com estadiamento IB evidenciado por histologia, acometimento da medula óssea e comprometimento clínico do estado geral. ${ }^{1}$

O National Cancer Institute recomenda a utilização

\section{REFERÊNCIAS}

1. Cassol L, Azevedo S. Linfomas não-Hodgkin em portadores da síndrome da imunodeficiência adquirida. Clinical \& Biomedical Research. 2004;24(2/3):18-27.

2. Coté TR, Biggar RJ, Rosenberg PS, Devesa SS, Percy C, Yellin FJ et al. Non Hodgkin's lymphoma among people with AIDS: Incidence, presentation and public health burden. Int J Cancer. 1997;73(5):645-50.

3. Mbulaiteye S, Biggar R, Goedert J, Engels E. Pleural and peritoneal lymphoma among People with AIDS in the United States. J Acquir Immune Defic Syndr. 2002;29(4):418-21.

4. Arzoo K, Bu X, Espina B, Seneviratne L, Nathwani B, Levine A. T-Cell lymphoma in HIV-infected patients. J Acquir Immune Defic Syndr. 2004;36(5):1020-7.

5. Chihuan GG, Butrón AF, Alejo RS, Frisancho O, Beltrán B. Linfoma plasmablástico: un caso con enfermedad rectal y compromiso de médula ósea en un paciente VIH positivo. Rev Gastroenterol Perú. 2014;34(4):347-50.

6. Castillo J, Pantanowitz L, Dezube BJ. HIV-associated plasmablastic lymphoma: lessons learned from 112 published cases. Am J Hematol. 2008;83(10):804-9. de etoposide, prednisona, vincristina, ciclofosfamida e doxorrubicina (esquema EPOCH) com o aumento gradativo da dose a ser aplicada. ${ }^{10} \mathrm{O}$ acompanhamento laboratorial do tratamento se faz com hemograma completo, dosagem de DHL e perfil bioquímico. Caso haja metástases, recomenda-se realizar tomografia computadorizada combinada com tomografia com emissão de positrons. ${ }^{11}$

\section{CONCLUSÃO}

Entre os linfomas não Hodgkin, o linfoma plasmablástico é a manifestação linfoproliferativa menos comum e que ocorre quase exclusivamente em pacientes com infecção pelo HIV. Esse relato é de importância para que se ampliem os diagnósticos diferenciais em pacientes com manifestações clínicas não usuais.

7. Sandler A, Kaplan L. Diagnosis and management of systemic non-hodgkin's lymphoma in hiv disease. Hematol Oncol Clin North Am. 1996;10(5):1111-24.

8. Kaplan L, Ai W. HIV-related Iymphomas: Clinical manifestations and diagnosis [Internet]. Uptodate. com. 2019 [acesso em 25 nov 2019]. Disponivel em: https://www.uptodate.com/contents/hiv-relatedlymphomas-clinical-manifestations-and-diagnosis.

9. Kaplan L, Rubenstein J. HIV-related Iymphomas: Primary central nervous system lymphoma [Internet]. Uptodate.com. 2019 [acesso em 25 nov 2019]. Disponivel em: https://www.uptodate.com/ contents/hiv-related-lymphomas-primary-centralnervous-system-lymphoma.

10. Little R, Pittaluga S, Grant N, Steinberg S, Kavlick $M$, Mitsuya $\mathrm{H}$ et al. Highly effective treatment of acquired immunodeficiency syndrome-related lymphoma with dose-adjusted EPOCH: impact of antiretroviral therapy suspension and tumor biology. Blood. 2003;101(12):4653-9.

11. Juweid M, Stroobants S, Hoekstra O, Mottaghy F, Dietlein M, Guermazi A et al. Use of positron emission tomography for response assessment of lymphoma: consensus of the Imaging Subcommittee of International Harmonization Project in Lymphoma. J Clin Oncol. 2007;25(5):571-8. 